\title{
Trombolisis cerebral en el adulto mayor. Caso clínico
}

\author{
Andrés Reccius, Sergio Illanes, Arturo Jaramillo, \\ José Luis Manterola, Violeta Díaz
}

\section{Thrombolysis for stroke in 93 years old female. Report of one case}

Most studies evaluating the usefulness of intravenous thrombolysis for acute stroke have excluded subjects aged over 80 years. Therefore there is no evidence to support or contraindicate this therapy in this age group. We report a 93 year-old female subjected to intravenous thrombolysis using tissue plasminogen activator ( $\mathrm{r}-\mathrm{tPA}$ ), according to the National Institute of Neurological Disorders protocol. The treatment was successful, there were no hemorrhagic complications and three months later, the patient was practically without any disability. Therefore age is not an absolute contraindication for intravenous thrombolysis in elderly subjects (Rev Méd Chile 2008; 136: 502-6).

(Key words: Aged, 80 and over; Stroke; Thrombolytic therapy)

Recibido el 16 de mayo, 2007. Aceptado el 24 de julio, 2007.

Unidad de Ataque Cerebrovascular, Departamento de Neurología-Neurocirugía. Hospital Clínico de la Universidad de Chile. Santiago, Chile.

$\mathrm{E}$ accidente cerebrovascular (ACV) constituye la segunda causa específica de muerte en nuestro país ${ }^{1}$, siendo el ACV isquémico el subtipo más frecuente $\left(69 \%\right.$ del total de los ACV) ${ }^{2}$. En Chile, la incidencia del infarto cerebral se estima en 87,3/100.000 habitantes, aumentando progresivamente con la edad, llegando a 762,5/100.000 en personas mayores a 85 años ${ }^{2}$. Tomando en cuenta el progresivo envejecimiento de nuestra población, se espera un notorio aumento de la incidencia del ACV. Por otro lado, el pronóstico del ACV se ensombrece a mayor edad ${ }^{3}$, lo que hace más necesario el uso de terapias efectivas contra esta enfermedad.

Correspondencia a: Dr. Sergio Illanes Díez. Santos Dumont $9992^{\circ}$ piso sector E. Fax: (56-2 7378546)

E mail: sergio.illanes@gmail.com
El único tratamiento específico aprobado para el infarto cerebral agudo es la trombolisis intravenosa con activador del plasminógeno tisular recombinante (t-PA) ${ }^{4}$. Sin embargo, el uso del t-PA se asocia a complicaciones hemorrágicas cerebrales y sistémicas, por lo que su uso se debiera limitar estrictamente al cumplimiento de protocolos validados y establecidos. El grupo de pacientes que ha mostrado un mayor beneficio clínico con esta terapia son aquellos de menor edad, en los que el t-PA administra antes de los 90 min de iniciado el ACV, que cursen con infartos cerebrales no masivos, sin hiperglicemia y que presenten un adecuado control de las cifras tensionales arteriales $^{4-8}$. Actualmente, muchos especialistas dudan del real beneficio del t-PA intravenoso en el grupo de mayor edad, basado fundamentalmente en la escasa experiencia publicada y el esperado mayor riesgo de complicaciones hemorrágicas asociadas. 
Para aportar en este debate, presentamos el caso de la primera paciente nonagenaria tratada en nuestro centro con terapia trombolítica intravenosa con t-PA.

\section{Caso clínico}

Paciente de sexo femenino de 93 años de edad, con antecedentes de hipertensión arterial crónica, usuaria de marcapaso definitivo debido a enfermedad del nodo sinusal, hipotiroidismo y cáncer de colon operado en su juventud. Vive sola, completamente autovalente, sin deterioro cognitivo.

El día del ingreso presentó de forma brusca un defecto motor del hemicuerpo derecho. Fue traída por familiares antes de una hora de evolución al Hospital Clínico de la Universidad de Chile (HCUCh). $\mathrm{Al}$ examen neurológico de ingreso destacaba una paciente vigil, orientada, disártrica, con una heminegligencia izquierda, hemiaopsia homónima izquierda, desviación de la mirada conjugada a derecha, hemiplejia izquierda disarmónica de predominio braquial e hipoestesia ipsilateral. El déficit neurológico estimado al ingreso empleando la escala del National Institute of Health Stroke Scale (NIHSS) fue de 14 puntos (donde un puntaje de 0 da cuenta de un examen neurológico normal, mientras que un puntaje de 42 representa a un paciente tetrapléjico y en coma), correspondiendo a un déficit neurológico moderado.

La tomografía computada de cerebro (TC) mostró signos precoces de isquemia cerebral a nivel lenticular derecho, con pérdida de diferenciación del límite lentículo-capsular y una señal sugerente de un trombo en el segmento proximal (M1) de la arteria cerebral media (ACM) derecha (Figura 1). La paciente fue ingresada a la Unidad de Tratamiento del Ataque Cerebrovascular (UTAC) del HCUCh, siguiendo el protocolo establecido para el manejo de estos pacientes en nuestro hospital.

No habiendo contraindicaciones para terapia trombolítica intravenosa, se realizó una trombolisis con t-PA a las $2 \mathrm{~h}$ de iniciados los síntomas del $\mathrm{ACV}$, de acuerdo a las pautas especificadas en el protocolo NINDS ${ }^{4}$, a una dosis de $0,9 \mathrm{mg} / \mathrm{kg}$, dividido el 10\% en un bolo de un minuto y el $90 \%$ restante en una infusión de $59 \mathrm{~min}$. La paciente evolucionó satisfactoriamente, mejorando su examen neurológico en forma progresiva, con un puntaje NIHSS de 7 al final del procedimiento.

La TC de control a las 24 h mostró un infarto consolidado no lacunar sólo en la región lenticular derecha, sin evidencia de daño isquémico en el resto del territorio irrigado por la ACM (Figura 2). No se observaron complicaciones hemorrágicas.

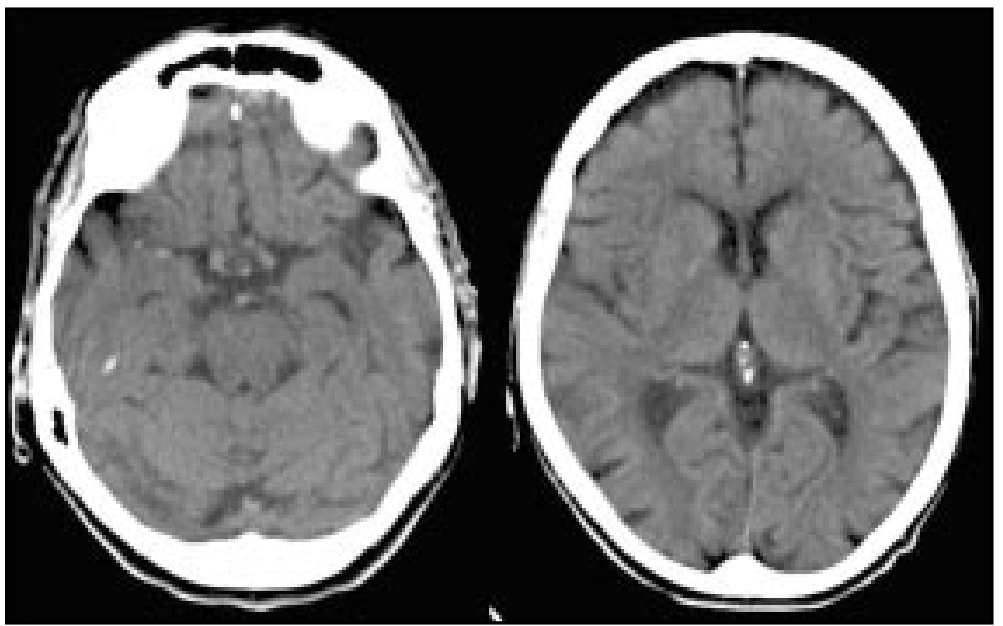

Figura 1. TC de cerebro sin contraste pretrombolisis intravenosa ( $1,5 \mathrm{~h}$ de evolución). Se observa signos precoces de isquemia cerebral a nivel lenticular derecho con pérdida de diferenciación del límite lentículocapsular y una señal sugerente de un trombo en el segmento proximal (M1) de la arteria cerebral media (ACM) derecha. 


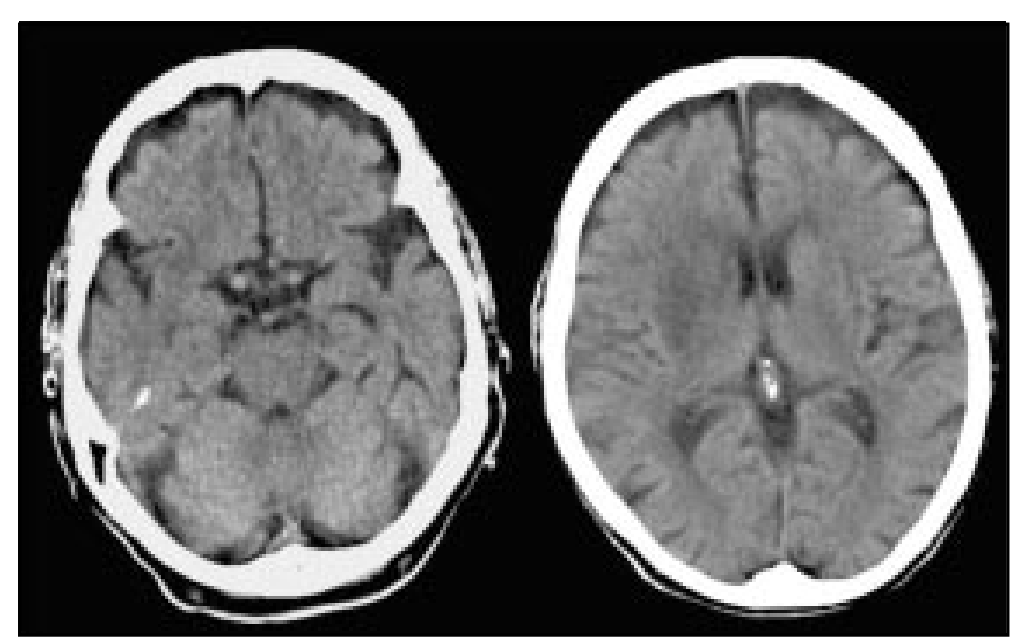

Figura 2. TC de cerebro sin contraste $24 \mathrm{~h}$ postrombolisis endovenosa. Se observa un infarto consolidado en la región lenticular derecha. No se observan complicaciones hemorrágicas.

Dentro del estudio etiológico destacaba una dilatación severa de la aurícula izquierda, con contraste espontáneo en su interior. Lo anterior, sumado al antecedente de la enfermedad del nodo sinusal y en ausencia de otras fuentes etiológicas, se planteó como causa probable del ACV un fenómeno cardioembólico, por lo que se inició tratamiento anticoagulante con heparina intravenosa y luego con acenocumarol oral. Dentro de su hospitalización no presentó complicaciones sistémicas de ningún tipo.

La paciente mejoró progresivamente, presentando al alta un valor en la escala NIHSS de 3 puntos. A los tres meses se encontraba completamente autovalente, con un valor en la escala modificada de Rankin de 1 punto, con mínimas alteraciones al examen neurológico y un puntaje NIHSS de 0 punto.

DisCUSIÓN

Según cifras oficiales del Instituto Nacional de Estadísticas, actualmente en Chile hay 278.803 personas mayores de 80 años de edad, lo que constituye $1,68 \%$ de la población. Las proyecciones indican que en 2020 llegaríamos a tener 456.788 personas mayores a 80 años en nuestro país, lo que a su vez representaría 2,46\% de nuestra población ${ }^{1}$. Esto supone un aumento importante en la incidencia del ACV, especialmente en los pacientes mayores.

Hasta ahora, no se dispone de estudios que evalúen el uso de t-PA intravenoso en este particular grupo etario de pacientes. El estudio NINDS ${ }^{4}$ que demostró la eficacia del t-PA intravenoso administrado antes de tres horas de evolución, no puso un límite superior en la edad de los pacientes, pero su promedio fue de 68 años (desviación estándar \pm 11 ) y sólo hubo 42 pacientes mayores a 80 años 9 . Las guías de recomendaciones terapéuticas internacionales ${ }^{10,11}$ y nacionales $^{12}$ no discuten específicamente acerca de la trombolisis en el grupo de mayor edad. En ellas tampoco se consideran algunas condiciones mórbidas asociadas a este grupo etario, como la demencia o la postración. En Europa, el estudio SITS-MOST ${ }^{13}$, que desarrolló la agencia europea regulatoria de productos medicinales, para la aprobación definitiva del t-PA endovenoso, excluyó explícitamente a los pacientes mayores de 80 años.

La controversia en cuanto al beneficio real de la terapia trombolítica intravenosa respecto de sus potenciales riesgos asociados en los pacientes mayores de 80 años, se sustenta en que algunos 
autores sostienen que la edad constituye un predictor de mal pronóstico en estos pacientes $6,7,14-16$, mientras que para otros, la edad no influiría significativamente en los resultados ${ }^{17-20}$. El análisis de subgrupos del estudio NINDS ${ }^{21}$ concluye que, a pesar de tener peor pronóstico, la mayor edad no contraindicaría el uso de t-PA, ya que si bien hubo un aumento discreto de la mortalidad (45\% versus 48\%), el número de pacientes con desenlace favorable (valor de la escala de Rankin modificada menor a 4, es decir, al menos capaces de caminar) también aumentó (14\% versus 30\%).

En definitiva, es altamente improbable que se realice un estudio aleatorio de trombolisis intravenosa contra placebo en pacientes mayores de 80 años diseñado específicamente para evaluar la seguridad y eficacia del tratamiento trombolítico en este grupo etareo. El uso rutinario de t-PA es difícil de justificar sólo en base a 42 pacientes del estudio NINDS ${ }^{4}$, más aún cuando los estudios observacionales muestran resultados conflictivos entre sí. Un estudio multicéntrico internacional actualmente en curso, el IST-3 (International

\section{REFERENCIAS}

1. Instituto Nacional de Estadísticas, (INE). Chile: Anuario de estadísticas vitales, 2003. http:// Www.ine.cl.

2. Lavados P, Sacks C, Prina L, Escobar A, Tossi C, ArAYA F ET AL. Incidence, 30-day case-fatality rate, and prognosis of stroke in Iquique, Chile: a 2year community-based prospective study (PISCIS project). Lancet 2005; 365: 2206-15.

3. Kammersgaard L, Jorgensen H, Reith J, Nakayama H, Pedersen P, Olsen T. Copenhagen Stroke Study. Short - and long-term prognosis for very old stroke patients. The Copenhagen Stroke Study. Age Ageing 2004; 33: 149-54.

4. Tissue plasminogen activator for acute ischemic stroke. The National Institute of Neurological Disorders and Stroke rt-PA Stroke Study Group. N Engl J Med 1995; 14: 333: 1581-7.

5. Lindsberg P, Kaste M. Thrombolysis for acute stroke. Curr Opin Neurol 2003; 16: 73-80.

6. Larrue V, von Kummer R, Muller A, Bluhmki E. Risk factors for severe hemorrhagic transformation in
Stroke Study 3$)^{22}$, busca responder algunas dudas respecto de la utilidad del t-PA en pacientes que no se ajustan al protocolo NINDS, y podría dar mayor información en el grupo de mayor edad.

En suma, creemos que el particular grupo de pacientes de más de 80 años de edad no debería ser excluido a priori de la opción de recibir terapia trombolítica considerando sólo su condición etaria, en especial si se cumplen con los criterios exigidos para la administración de t-PA. En nuestra opinión, creemos que lo más aconsejable en estos casos es tomar una decisión individual, evaluando su aplicabilidad del tratamiento y sus riesgos caso a caso, tomando en cuenta diversas variables como la expectativa de vida, condición mórbida previa, aspectos psicosociales, así como también la opinión informada del enfermo y su familia. Hay que tener en cuenta que la edad avanzada es un factor independiente de mal pronóstico del ACV, pero que por sí misma no contraindica su aplicabilidad. El buen resultado observado en nuestra paciente es un ejemplo de factibilidad del uso de la terapia trombolítica intravenosa en pacientes mayores.

ischemic stroke patients treated with recombinant tissue plasminogen activator: a secondary analysis of the European-Australasian Acute Stroke Study (ECASS II). Stroke 2001; 32: 438-41.

7. Tanne D, Kasner S, Demchuk A, Koren-Morag N, Hanson S, Grond M et al. Markers of Increased Risk of Intracerebral Hemorrhage After Intravenous Recombinant Tissue Plasminogen Activator Therapy for Acute Ischemic Stroke in Clinical Practice. The Multicenter rt-PA Acute Stroke Survey. Circulation 2002; 105: 1679-85.

8. Albers G, Bates V, Clark W, Bell R, Verro P, Hamilton S. Intravenous tissue-type plasminogen activator for treatment of acute stroke: the Standard Treatment with Alteplase to Reverse Stroke (STARS) study. JAMA 2000; 283: 1145-50.

9. Wardlaw JM, Sandercock PA, Berge E. Thrombolytic therapy with recombinant tissue plasminogen activator for acute ischemic stroke: where do we go from here? A cumulative meta-analysis. Stroke 2003; 34: 1437-42.

10. Adams HP Jr, del Zoppo G, Alberts M, Bhatt D, Brass L, Furlan A et al. Guidelines for the early 
management of adults with ischemic stroke: a guideline from the American Heart Association/ American Stroke Association Stroke Council, Clinical Cardiology Council, Cardiovascular Radiology and Intervention Council, and the Atherosclerotic Peripheral Vascular Disease and Quality of Care Outcomes in Research Interdisciplinary Working Groups: the American Academy of Neurology affirms the value of this guideline as an educational tool for neurologists. Stroke 2007; 38: 1655-711.

11. Hacke W, Kaste M, Bogousslavsky J, Brainin M, Chamorro A, European Stroke Initiative Executive Committee and the EUSI Writing Committee. European Stroke Initiative Recommendations for Stroke Management-update 2003. Cerebrovasc Dis 2003; 16: 311-37.

12. Guías de práctica clínica para diagnóstico y manejo del ataque cerebrovascular agudo. Grupo de trabajo en enfermedades cerebrovasculares Sociedad Chilena de Neurología, Psiquiatría y Neurocirugía, Comisión Ministerial de Guías de Práctica Clínica de Manejo de Enfermedades Cerebrovasculares. http://www.sonepsyn.cl/pdf/ guias.pdf.

13. Wahlgren N, Ahmed N, Davalos A, Ford G, Grond M, HACKE W ET AL. Thrombolysis with alteplase for acute ischaemic stroke in the Safe Implementation of Thrombolysis in Stroke-Monitoring Study (SITS-MOST): an observational study. Lancet 2007; 369: 275-82.

14. Mouradian M, Senthilselvan A, Jickling G, Mc Combe J, Emery D, DeAn N ET al. Intravenous rt-PA for acute stroke: comparing its effectiveness in younger and older patients. J Neurol Neurosurg Psychiatry 2005; 76: 1234-7.
15. Berrouschot J, Rother J, Glahn J, Kucinski T, Fiehler J, Thomalla G. Outcome and severe hemorrhagic complications of intravenous thrombolysis with tissue plasminogen activator in very old $(\geq 80$ years) stroke patients. Stroke 2005; 36: 2421-5.

16. van Oostenbrugge R, Hupperts R, Lodder J. Thrombolysis for acute stroke with special emphasis on the very old: experience from a single Dutch centre. J Neurol Neurosurg Psychiatry 2006; 77 : 375-7.

17. Saposnik G, Young B, Silver B, Di Legge S, Webster F, Beletsky V eT AL. Lack of improvement in patients with acute stroke after treatment with thrombolytic therapy: predictors and association with outcome. JAMA 2004; 292: 1839-44.

18. Demchuk A, Tanne D, Hill M, Kasner S, Hanson S, and the Multicentre tPA Stroke Survey Group. Predictors of good outcome after intravenous tPA for acute ischemic stroke. Neurology 2001; 57 : 474-80.

19. Tanne D, Gorman M, Bates V, Kasner S, Scott P, Verro P ET AL. Intravenous tissue plasminogen activator for acute ischemic stroke in patients aged 80 years and older: the tPA stroke survey experience. Stroke 2000; 31: 370-5.

20. Simon J, Sandler D, Pexman J, Hill M, Buchan A. Calgary Stroke Programme. Is intravenous recombinant tissue plasminogen activator (rt-PA) safe for use in patients over 80 years old with acute ischaemic stroke? - The Calgary experience. Age Ageing 2004; 33: 143-9.

21. The NINDS t-PA Stroke Study Group. Generalized efficacy of t-PA for acute stroke. Subgroup analysis of the NINDS t-PA Stroke Trial. Stroke 1997; 28: 2119-25.

22. www.ist3.com 